\title{
Canada's National Collaborating Centres: Facilitating evidence-informed decision-making in public health
}

\author{
Alejandra Dubois ${ }^{1 \star}$, Mélanie Lévesque $^{1}$
}

\begin{abstract}
Although evidence-informed decision-making is fundamental to public health, it is challenging in practice as there is a continual burgeoning of both evidence and emerging issues, which public health professionals need to address at local, regional and national levels. One way that Canada has addressed this perennial challenge is through its six National Collaborating Centres (NCCs). The NCCs for Public Health were created to promote and support the use of scientific research and other knowledge to strengthen public health practice, programs and policies in Canada. The NCCs identify knowledge gaps, foster networks across sectors and jurisdictions and provide the public health system with an array of evidence-informed resources and knowledge translation services. Each centre is hosted in academic or government organizations across Canada and focuses on a specific public health priority: Determinants of Health; Environmental Health; Healthy Public Policy; Indigenous Health; Infectious Diseases; and Knowledge Translation Methods and Tools. Since their launch in 2005, the NCCs have undergone two federal evaluations, the results of which clearly demonstrate their significant contribution to evidence-informed decision-making in public health in Canada, while identifying some opportunities for future growth. The NCCs successfully help to bridge the gaps between evidence, policy and practice and facilitate the implementation of evidence in multiple, often complex, settings.
\end{abstract}

This work is licensed under a Creative Commons Attribution 4.0 International License.

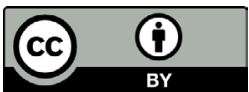

Affiliation

${ }^{1}$ Office of the Chief Science Officer, Public Health Agency of Canada, Ottawa, ON

*Correspondence: alejandra dubois@canada.ca

Suggested citation: Dubois A, Lévesque M. Canada's National Collaborating Centres: Facilitating evidenceinformed decision-making in public health. Can Commun Dis Rep 2020;46(2/3):31-5.

http://doi.org/10.14745/ccdr.v46i23a02

Keywords: Public health, networks, knowledge synthesis, knowledge translation, health equity, determinants of health, evidence-informed practice, evidence-informed decision-making

\section{Introduction}

A hallmark of public health in Canada and around the world is evidence-informed decision-making (EIDM) (1). In light of the fact that new knowledge is being continuously generated, there is an ongoing need in public health to synthesize this new evidence in the context of what is already known, incorporate it into the development and implementation of policy and practice and evaluate its application to ensure the desired outcomes are achieved. Although EIDM is fundamental to public health, it is challenging in practice. The World Health Organization has recognized that real-world implementation of evidence-based interventions is "one of the greatest challenges ... [for] the global health community" (1). The challenges for public health in Canada include the following:

1. Decision makers may not have access to new evidence as it becomes available
2. Not all public health professionals have the knowledge, skills, or resources to undertake knowledge syntheses and knowledge transfer activities which are adapted to the needs of decision makers situated in various contexts

3. Public health organizations may lack the infrastructure to support such activities (2)

One way that Canada has addressed these perennial challenges is to develop the six National Collaborating Centres (NCCs), known collectively as the NCCs for Public Health program (NCCPH; www.nccph.ca). The NCCs were established in 2005 as part of the federal government's commitment to renew and strengthen public health in Canada (3). They were designed to promote and support the use of scientific research and other knowledge to strengthen public health practice, programs and policies in Canada. The NCCs have been described as a unique 
network of "knowledge brokers" (4) who collectively "identify knowledge gaps, foster networks and provide the public health system with an array of evidence-based resources ... and knowledge translation services" (5).

It is a tall order. The NCCs carry out their mission by fostering collaboration and networking among diverse stakeholders and drawing on regional, national and international expertise. They work with a wide range of organizations and across jurisdictions to create opportunities to learn from each other and to work together. For example, the NCCs partner with organizations such as the Pan-Canadian Public Health Network Council, the formal network that links all 13 provincial/territorial governments with the Public Health Agency of Canada and reports to the Conference of Federal/Provincial/Territorial Deputy Ministers of Health (6).

The purpose of this article is to describe what the NCCs do, identify their current priorities and topic areas for 2020 and beyond, and describe how they are able to facilitate the bridging of evidence and other knowledge systems into policy and practice.

\section{The National Collaborating Centres for Public Health}

Funded by the Public Health Agency of Canada and located across the country (Figure 1), each of the six NCCs focuses on a specific public health area: Determinants of Health, Environmental Health, Healthy Public Policy, Indigenous Health, Infectious Diseases, and Knowledge Translation Methods and Tools. Each NCC is hosted by an academic institution or government-based organization.

The NCCs synthesize and disseminate high-quality evidence and knowledges, foster collaboration among diverse stakeholders, and support public health professionals, policymakers and decision makers in using evidence-informed knowledge to improve health outcomes for Canadians. The NCCs turn research and other information into knowledge products tailored to specific audiences, contextualized to their settings and in both official languages. These include products such as guidance documents, reports, evidence reviews, fact sheets, and case studies.

\section{Figure 1: National Collaborating Centres for Public Health}

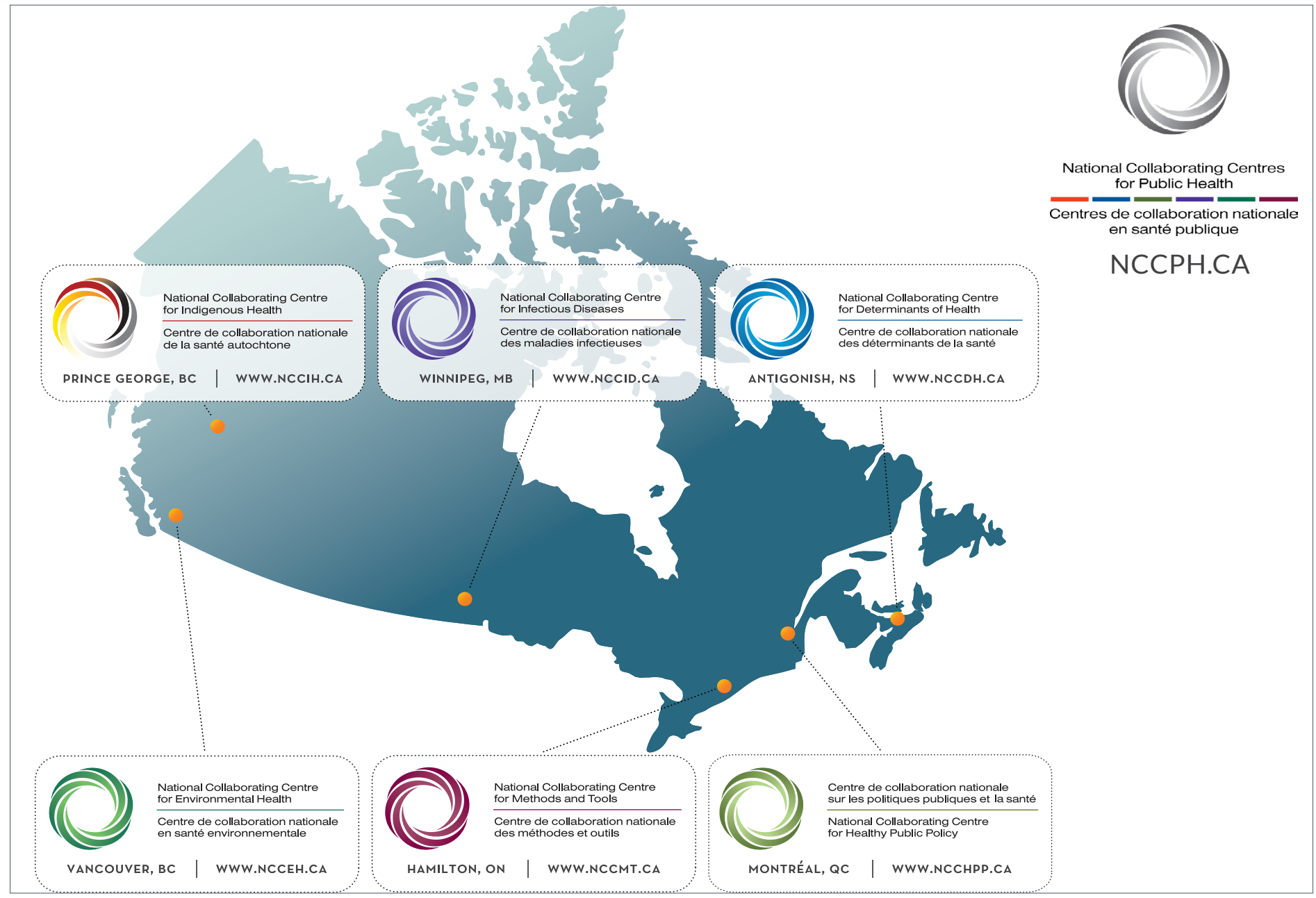


Each NCC has an expert advisory board for strategic orientation and advice. Priorities are established through a variety of strategies, including convening national gatherings, supporting and participating in networks and committees, administering surveys, conducting environmental scans and literature reviews, interviewing key informants, evaluating activities and resources, partnering with Indigenous leaders and organizations and working with governments at all levels. As described in a recent NCCPH document presented to the Pan-Canadian Public Health Network Council (unpublished document, National Collaboration Centres for Public Health, 2019), current priorities, strategies and key focus areas of each NCC are described in the following section.

\section{NCC for Determinants of Health}

The NCC for Determinants of Health (NCCDH) is hosted by St. Francis Xavier University in Antigonish, Nova Scotia. The $\mathrm{NCCDH}$ focuses on three priority areas: addressing the structural drivers of inequity; supporting a "culture of equity" in public health; and promoting action on the factors that influence health equity, the social determinants of health. Strategies for doing this include making health equity an explicit goal in organizations, programs and policies, promoting public health roles to advance health equity, supporting the application of promising equityoriented knowledge and practices, facilitating networks and knowledge exchange and influencing knowledge translation practices to support action on health equity.

\section{NCC for Environmental Health}

The NCC for Environmental Health (NCCEH) is located at the British Columbia Centre for Disease Control, in Vancouver, British Columbia. The NCCEH has three priorities. The first is to raise awareness and increase understanding of 1) existing and emerging environmental threats and benefits and 2) how to mitigate these threats and optimize the benefits. The second is to translate and highlight research that informs the effective practice of environmental health. The third involves bringing together the aggregate experience of environmental health practitioners across Canada to inform practice that is effective and attuned to the evolving orientation of public health. Key areas include 1) both natural and built environments, 2) the changing climate, emergency preparedness and response and 3) resources to support public health inspection, protection and policy-making.

\section{NCC for Healthy Public Policy}

The NCC for Healthy Public Policy (NCCHPP) is hosted by the Institut national de santé publique du Québec in Montreal, Quebec. The NCCHPP has three priorities. First, to support the development of competencies and organizational capacity in policy analysis. Second, to support the implementation of intersectoral approaches to promote healthy public policies. Finally, to develop policy approaches for emerging issues in public health. Priority topics include public policy analysis, health in all policy, health impact assessment, climate change, population mental health and well-being, public health ethics, health inequalities as well as knowledge sharing.

\section{NCC for Indigenous Health}

The NCC for Indigenous Health $(\mathrm{NCClH})$ is based at the University of Northern British Columbia, in Prince George, British Columbia. The $\mathrm{NCClH}$ has two priorities. The first priority is to increase understanding and application of Indigenousinformed evidence on First Nations, Inuit and Métis health across their lifespan to support public health policy, practice and program decision-making. The second is to foster partnerships, collaborations and networks to mobilize Indigenous-informed evidence across sectors and jurisdictions to support Indigenous health equity. The $\mathrm{NCClH}$ health pillars include emerging priorities in public health, social determinants of health, child, youth and family health and Indigenous knowledge and public health.

\section{NCC for Infectious Diseases}

The NCC for Infectious Diseases (NCCID) is based at the University of Manitoba, in Winnipeg, Manitoba. The NCCID set three priorities for the upcoming years. The first is the support of public health responses to infectious diseases among migrants and mobile populations. The second is to address inequities in public health responses to communicable diseases in rural and remote communities. The third is to support opportunities for using big data for infectious disease surveillance, prevention, control and monitoring. Topics include HIV and sexually transmitted and bloodborne infections (STBBIs), tuberculosis, stigma, locally and culturally appropriate interventions, the Notifiable Diseases Database, antimicrobial resistance and stewardship, and climate change and vector-borne illnesses. The NCCID supports topic-specific networks (such as the STBBI Network and the past AMS Canada), and facilitates two or more national gatherings for knowledge exchange each year.

\section{NCC for Methods and Tools}

The NCC for Methods and Tools (NCCMT) is located at McMaster University, in Hamilton, Ontario. The NCCMT has three priorities. The first is to support evidence-informed decision-making in public health in Canada. The second is to make easily accessible, and, where gaps exist, to develop, methods and tools that facilitate increased capacity for evidenceinformed decision-making. The third is to facilitate and support organizational change among public health organizations. The NCCMT develops resources to build organizational capacity, knowledge and skills related to EIDM across the broad spectrum of public health services in Canada, thus supporting better decision and policy-making across the major domains of public health. The NCCMT engages with regional, national and international networks and partners, across public health practice, education, research and knowledge translation, to identify gaps in knowledge and its application and collaborate to develop new tools and resources. 


\section{Discussion}

For the nearly 15 years since their creation, the NCCs have demonstrated a proven track record for supporting and responding to the needs of public health with evidence, knowledge systems and building networks. Their biggest challenges are choosing what to work on, with whom, where and how. Indeed, public health includes a diverse interdisciplinary workforce, a wide range of programs and services and a large network of intersecting partners and stakeholders including community members and all levels of government.

To ascertain the effectiveness of the NCCPH program, two federal evaluations were conducted: the first one spanned from 2008 to early 2014 (7); and the second from 2014 to September 2018 (8). The latest evaluation report concluded that the NCCs are credible go-to sources on numerous public health issues, producing a wide range of high-quality knowledge translation products. The report identified many examples of NCC contributions to decision and policy-making in the public health field. It also indicated that the "NCCs' ability to collaborate on different initiatives and to network with different partners across the public health system is seen as one of the most valued capabilities of the Centres" (8).

The 2018 evaluation found that there were many examples of the contributions that NCCs have made to address emerging public health issues. It did, however, identify the need for flexibility to address emerging issues and the potential value of more collaboration with Public Health Agency of Canada in order to leverage the respective organizations' knowledge, resources and networks.

The current priorities, strategies and key areas of the NCCs are consistent with many of the macro trends in public health today: climate change, Health in All Policies, structural determinants of health inequities, big data, and demographic transitions (such as migrant and mobile populations) (9). The NCCs work is also consistent with the recent Public Health 3.0 approach advocated by the United States Department of Health and Human Services, which emphasizes "cross-sector collaboration and environmental, policy, and systems-level actions that directly affect the social determinants of health" (10).

The Public Health Agency of Canada recognizes the contribution of the NCCs and reaffirms its commitment to strengthening public health capacity through science, knowledge and EIDM. The NCCs are a foundational pillar of the Canadian public health infrastructure and a very valuable asset to protect and to benefit from.

\section{Conclusion}

There is an ongoing need for knowledge translation services to make evidence accessible and useful to public health professionals and organizations as well as all levels of government to advance national public health priorities. In addition, "in Canada's multijurisdictional health system, there continues to be a need to foster networks across the system. NCCs tend to occupy a unique niche focused on translating evidence and knowledge in a very practical manner to support public health professionals and organizations across the country" (8). The NCCs continue to fill a critical role in public health in Canada by helping to identify knowledge gaps and to bridge the divide between evidence, policy and practice. The NCCs have successfully demonstrated the ability to support the implementation of evidence in multiple, often complex settings, and will continue to take a leading role in Canada's public health system.

\section{Authors' statement}

$A D$ - Original conception, review of drafts and final version $\mathrm{ML}$ - Contribution to design, substantive input, review of drafts

\section{Conflict of interest}

None.

\section{Acknowledgements}

Many thanks to key members in each of the National Collaborating Centres for Public Health for reviewing earlier drafts of this article and for providing input over the holiday season. Our thanks to Dr. Patricia Huston who contributed to the initial structure and design of the manuscript.

\section{References}

1. Peters DH, Tran NT, Adam T. Implementation Research in Health: A Practical Guide. Alliance for Health Policy and Systems Research, World Health Organization. Geneva (CH): WHO; 2013. https://apps.who.int/iris/bitstream/hand le/10665/91758/9789241506212_eng.pdf;jsessionid=95637 C59A3DFE73AF3EDE87C986C2779? sequence $=1$

2. Peirson L, Ciliska D, Dobbins M, Mowat D. Building capacity for evidence informed decision making in public health: a case study of organizational change. BMC Public Health 2012 Feb;12:137. DOI PubMed 
3. Medlar B, Mowat D, Di Ruggiero E, Frank J. Introducing the National Collaborating Centres for Public Health. CMAJ 2006 Aug;175(5):493-4. DOl PubMed

4. McAteer J, Di Ruggiero E, Fraser A, Frank JW. Bridging the academic and practice/policy gap in public health: perspectives from Scotland and Canada. J Public Health (Oxf) 2019 Sep;41(3):632-7. DOI PubMed

5. National Collaborating Centres for Public Health. About Us (Accessed 2020-01-02). https://nccph.ca/about-us/

6. Pan-Canadian Public Health Network. About the Pan-Canadian Public Health Network. PHN (Accessed 2019-12-31). http://www.phn-rsp.ca/network-eng.php

7. Health Canada and Public Health Agency of Canada. Evaluation of the National Collaborating Centres for Public Health program 2008-2009 to 2013-2014. Ottawa (ON):PHAC (Accessed 2020-01-02).

https://www.canada.ca/en/public-health/corporate/ mandate/about-agency/office-evaluation/evaluation-reports/ evaluation-national-collaborating-centres-public-healthprogram-2008-2009-2013-2014.html
8. Health Canada and Public Health Agency of Canada. Evaluation of the National Collaborating Centres for Public Health program 2014-2015 to 2018-2019. Ottawa (ON):PHAC; p. 29 (Accessed 2020-01-02). https://www.canada.ca/en/public-health/corporate/ transparency/corporate-management-reporting/ evaluation/2014-2015-2018-2019-evaluation-report-nationalcollaborating-centres-public-health-program.html

9. Erwin PC, Brownson RC. Macro Trends and the Future of Public Health Practice. Annu Rev Public Health 2017 Mar;38:393-412.

DOI PubMed

10. DeSalvo KB, O'Carroll PW, Koo D, Auerbach JM, Monroe JA. Public Health 3.0: time for an Upgrade. Am J Public Health 2016 Apr;106(4):621-2. DOI PubMed 